\title{
Acteoside attenuates hydrogen peroxide-induced injury of retinal ganglion cells via the CASC2/miR-155/mTOR axis
}

\author{
Xiaoting Xi", Jia Ma", Qianbo Chen, Xuewei Wang, Yuan Xia, Xuewei Wen, Jin Yuan, Yan Li \\ Department of Ophthalmology, The First Affiliated Hospital of Kunming Medical University, Kunming, China \\ Contributions: (I) Conception and design: Y Li; (II) Administrative support: Y Li; (III) Provision of study materials: X Xi, J Ma, Q Chen, X Wang; \\ (IV) Collection and assembly of data: X Xi, J Ma, Y Xia, X Wen; (V) Data analysis and interpretation: X Xi, M Ji, J Yuan; (VI) Manuscript writing: All \\ authors; (VII) Final approval of manuscript: All authors. \\ "These authors contributed equally to this work. \\ Correspondence to: Yan Li. Department of Ophthalmology, The First Affiliated Hospital of Kunming Medical University, No. 295 Xichang Road, \\ Kunming 650031, China. Email: li_yan_km@163.com.
}

Background: Loss of retinal ganglion cells (RGCs), which eventually leads to optic nerve atrophy and vision loss, is the main cause of glaucoma and traumatic optic neuropathy. Acteoside is the effective component of Yunnan Kudingcha, which has been reported to exert neuroprotective effects and protects RGCs from injury. However, the underlying mechanisms of acteoside in RGC injury remain largely elusive.

Methods: Human RGCs was treated with hydrogen peroxide $\left(\mathrm{H}_{2} \mathrm{O}_{2}\right)$. The expression of miR-155 and lncRNA CASC2 in RGC-5 cells was measured by RT-qPCR. The viability of RGCs was determined by the MTT assay. Flow cytometry and TUNEL staining were used to detect cell apoptosis. The malondialdehyde (MDA) content and superoxide dismutase (SOD) activity were determined using ELISA kits. The mTOR and autophagic proteins were measured by western blot.

Results: We identified the expression of miR-155 was upregulated in $\mathrm{H}_{2} \mathrm{O}_{2}$-treated RGCs, and enhanced miR-155 promoted RGC autophagy and apoptosis. Acteoside administration reduced miR-155 expression and abolished miR-155-mediated RGC injury. The expression of CASC2 was decreased in $\mathrm{H}_{2} \mathrm{O}_{2}$-treated RGCs. Acteoside administration could increase CASC2 expression and CASC2 overexpression reverses the effect of miR-155 overexpression on acteoside treatment-RGCs. Mechanistically, we discovered that highly expressed miR-155 promoted RGC autophagy and apoptosis via the mTOR pathway. In addition, acteoside attenuated RGC autophagy and apoptosis via the miR-155/mTOR axis. Together, these results identify a mechanism by which acteoside attenuates $\mathrm{H}_{2} \mathrm{O}_{2}$-induced RGC apoptosis and autophagy via the CASC2/ miR-155/mTOR axis.

Conclusions: Acteoside protects RGC-5 cells against $\mathrm{H}_{2} \mathrm{O}_{2}$-induced cell injury via the CASC2/miR-155/ mTOR axis. These results provide new insights for early medical interventions in patients with glaucoma.

Keywords: Retinal ganglion cells; acteoside; mTOR; apoptosis; autophagy

Submitted Aug 12, 2021. Accepted for publication Dec 08, 2021.

doi: 10.21037/atm-21-5630

View this article at: https://dx.doi.org/10.21037/atm-21-5630

\section{Introduction}

Glaucoma, a neurodegenerative eye disease that leads to blindness, is attributed to retinal ganglion cell (RGC) loss (1). Emerging studies have demonstrated that Chinese traditional medicines such as Lycium barbarum, Ginkgo biloba (2), and tetramethylpyrazine (3) play critical roles in treating glaucoma. Acteoside, as an effective component of Yunnan Kudingcha, has been reported to attenuate the apoptosis of RGCs and glaucoma-induced optic atrophy (4). However, the underlying mechanism of acteoside in RGC apoptosis is still unclear.

MicroRNAs (miRNAs) are a class of conserved small 
non-coding RNAs that function as key players in glaucoma development, diagnosis, and treatment (5-7). In addition, the role of miRNAs in the activity regulation of RGCs has been well characterized. For example, miR-93-5p modulates NMDA-induced RGC autophagy via the AKT/mTOR pathway in glaucoma (8). Furthermore, miR-141-3p inhibits RGC apoptosis in glaucoma mice (9), and inhibition of miR-149 could suppress RGC apoptosis in glaucoma mice (10). A study also indicated that miR-155 is induced in neurodegenerative diseases including glaucoma (11). Moreover, miR-155 has been reported to inhibit retinal function (12). These studies suggest that miR-155 may be involved in glaucoma development.

It is recognized that the mTOR signaling pathway contributes to the activation of RGCs $(13,14)$. In addition, mounting evidence demonstrates that miR-155 can modulate pathological states including cardiomyopathy (15), cancer (16), and alcoholic liver disease (17) via targeting the mTOR signaling pathway. Yet, the function of the miR$155 / \mathrm{mTOR}$ axis in glaucoma is unknown.

In the present study, we established hydrogen peroxide $\left(\mathrm{H}_{2} \mathrm{O}_{2}\right)$-induced RGC injury and determined the role of miR-155 in acteoside-mediated glaucoma treatment. miR151 was induced in $\mathrm{H}_{2} \mathrm{O}_{2}$-treated RGCs. Inhibition of miR155 alleviated $\mathrm{H}_{2} \mathrm{O}_{2}$-induced RGC apoptosis and autophagy. Acteoside not only attenuated apoptosis and autophagy of RGCs, but also suppressed miR-155 expression. Moreover, we confirmed that acteoside protected RGCs from injury via miR-155. We further demonstrated that miR-155 could directly target mTOR, and the mTOR signaling pathway was responsible for acteoside/miR-155-mediated RGC apoptosis and autophagy. Thus, our study revealed a novel mechanism by which acteoside protected against RGC injury via the miR-155/mTOR axis.

We present the following article in accordance with the MDAR reporting checklist (available at https://dx.doi. org/10.21037/atm-21-5630).

\section{Methods}

\section{Cell culture and treatment}

RGCs (RGC-5 cell line) were obtained from American Type Culture Collection (ATCC, YB-ATCC-7491) and maintained in Dulbecco's modified Eagle medium (DMEM, Invitrogen) supplemented with $10 \%$ heat-inactivated fetal bovine serum (FBS, Gibco) and 1\% penicillin/streptomycin. RGCs were cultured in a humidified atmosphere of $95 \%$ air and $5 \% \mathrm{CO}_{2}$ at $37{ }^{\circ} \mathrm{C}$. To establish oxidative stress injury in RGCs, the RGCs were treated with $200 \mu \mathrm{M} \mathrm{H}_{2} \mathrm{O}_{2}$ as previously described (18). Acteoside (purity $\geq 98 \%$, Sigma Chemical Co., MO, USA) was used to protect RGC5 cells against $\mathrm{H}_{2} \mathrm{O}_{2}$-induced cell injury with $3 \mathrm{mg} / \mathrm{mL}$ concentration as our previous reported (19).

\section{Cell transfection}

The miR-155 inhibitor, miR-155 mimic, CASC2 overexpression vector (oe-CASC2) and corresponding control (NC inhibitor, NC mimic and oe-NC) were synthesized and purified by RiboBio. RGCs were transfected with the oe-CASC2 $(2 \mu \mathrm{g} / \mu \mathrm{L})$, oe-NC $(2 \mu \mathrm{g} / \mu \mathrm{L})$, miR-155 inhibitor $(50 \mathrm{nM})$, NC inhibitor $(50 \mathrm{nM})$, miR155 mimic (100 $\mathrm{nM})$ and NC mimic (100 $\mathrm{nM})$ using Lipofectamine 2000 reagent (Invitrogen) according to the manufacturer's instruction.

\section{Cell viability measurement}

The viability of RGCs was measured by the MTT assay using the MTT Cell Proliferation and Cytotoxicity Assay Kit (C0009S, Beyotime). Briefly, RGC-5 cells were seeded in a 96-well plate $\left(2 \times 10^{5} /\right.$ well $)$ and treated with $\mathrm{H}_{2} \mathrm{O}_{2}$ for $24 \mathrm{~h}$. Subsequently, $10 \mu \mathrm{L}$ MTT solution was added to RGCs and incubated at $37^{\circ} \mathrm{C}$ for $4 \mathrm{~h}$. The absorbance at $570 \mathrm{~nm}$ was measured.

\section{Cell apoptosis measurement}

The indicated RGCs were treated with $\mathrm{H}_{2} \mathrm{O}_{2}$ for $24 \mathrm{~h}$ and subjected to flow cytometry analysis using the Annexin V-FITC Apoptosis Detection Kit (C1062S, Beyotime) for cell apoptosis detection. Briefly, RGCs were harvested and mixed with $5 \mu \mathrm{L}$ Annexin V-FITC and $10 \mu \mathrm{L}$ propidium iodide (PI). After incubation for $30 \mathrm{~min}$, RGCs were analyzed with flow cytometry.

\section{Western blot assay}

Total protein was isolated from RGCs using RIPA Lysis Buffer (P0013B, Beyotime) and the concentration of protein was measured by the BCA Protein Assay Kit (P0012, Beyotime). Equal amounts of proteins were separated by SDS-polyacrylamide gel electrophoresis and transferred to PVDF membranes (ISEQ00010, Millipore). After blocking with non-fat milk, the membranes were incubated with the 
following primary and secondary antibodies: $\mathrm{mTOR}$ (ab2732, Abcam, 1:1,000), LC3 (ab2775, Cell Signaling Technology, 1:2,000), and p62 (ab91526, Abcam, 1:2,000). GAPDH (ab9485, Abcam, 1:5,000) served as the loading control.

\section{RNA isolation and quantitative real-time PCR (RT-qPCR) assay}

Total RNA was extracted from RGCs using Trizol reagent (15596026, Invitrogen), and cDNA was synthesized using the miRNA 1st Strand cDNA Synthesis Kit (MR10101, Vazyme). RT-qPCR was then carried out with SYBR Green Premix (DRR041A, Takara) and analyzed with the $2^{-\Delta \Delta \mathrm{Ct}}$ method (20). The cycling conditions were as follows: $50{ }^{\circ} \mathrm{C}$ for $2 \mathrm{~min}, 95^{\circ} \mathrm{C}$ for $10 \mathrm{~min}$, followed by 40 cycles of $95{ }^{\circ} \mathrm{C}$ for $15 \mathrm{~s}$ and $60{ }^{\circ} \mathrm{C}$ for $1 \mathrm{~min}$. The primers used in this study were as follows: miR-155, forward: 5'-GGAGGTTAATGCTAATCGTGATAG-3'; reverse: 5'-GTGCAGGGTCCGAGGT-3' . U6, forward: 5'-TCGGCAGCACATATACTAA-3'; reverse: 5'-CGCTTCACGAATTTGCGTGT-3'. U6 was used as the endogenous control.

\section{Malondialdebyde (MDA) content and superoxide dismutase (SOD) activity assays}

The MDA content and SOD activity in RGCs were determined using commercially available ELISA kits (Jiancheng Bioengineering Institute, Nanjing, China) according to the manufacturer's instructions. Absorbance was determined using a microplate spectrophotometer (BioTeke).

\section{TUNEL staining}

RGCs were treated or transfected and then cultured $48 \mathrm{~h}$. After rising twice with PBS, the cells were fixed with $4 \%$ paraformaldehyde for $15 \mathrm{~min}$ at $37^{\circ} \mathrm{C} .1 \times 10^{4}$ cells were prepared in 96-well plates and then subjected to ClickiTTM Plus TUNEL Assay Kit (ThermoFisher Scientific, Waltham, MA) according to the manufacturer's instructions. Following DAPI staining, the apoptotic cells were measured by using Nikon Eclipse 80i microscope (Nikon Corporation).

\section{Statistical analysis}

All data are presented as the mean \pm SE from at least 3 biological replicates, and the differences between any
2 groups or multiple groups were compared using unpaired $t$-tests.

\section{Results}

\section{MiR-155 is induced upon $\mathrm{H}_{2} \mathrm{O}_{2}$ treatment of $\mathrm{RGC-5}$ cells}

In order to determine the role of miR-155 in RGC-5 cell injury. We established RGCs oxidative stress injury model, as previous reported that RGC-5 cells were treated with different concentrations of $\mathrm{H}_{2} \mathrm{O}_{2}$ for $12 \mathrm{~h} \mathrm{(21),16} \mathrm{h} \mathrm{(3)} \mathrm{and}$ $24 \mathrm{~h}$ (18), which $200 \mu \mathrm{M} \mathrm{H} \mathrm{O}_{2}$ significantly reduced RGC5 cells viability at 12,16 and $24 \mathrm{~h}$. Thus, we selected 200 $\mu \mathrm{M} \mathrm{H}_{2} \mathrm{O}_{2}$ treated RGC-5 cells. As shown in Figure 1A, $\mathrm{H}_{2} \mathrm{O}_{2}$ treatment inhibited RGC-5 cell viability. The content of MDA, an oxidative stress marker, was elevated, while the activity of SOD, an anti-oxidative enzyme, was reduced, in the $\mathrm{H}_{2} \mathrm{O}_{2}$ treatment (Figure $1 B, 1 C$ ). $\mathrm{H}_{2} \mathrm{O}_{2}$ treatment also promoted RGC-5 cell autophagy (Figure $1 D$ ) and apoptosis (Figure $1 E, 1 F$ ). In addition, miR-155 expression was induced in $\mathrm{H}_{2} \mathrm{O}_{2}$-treated RGC-5 cells (Figure $1 G$ ).

\section{MiR-155 inbibition alleviates $\mathrm{H}_{2} \mathrm{O}_{2}$-induced $\mathrm{RGC}-5$ cell injury}

To further identify the function of miR-155 in $\mathrm{H}_{2} \mathrm{O}_{2}$ induced RGC-5 cell injury, we transfected cells with an miR-155 inhibitor or NC inhibitor as the control (Figure $2 A$ ). After $\mathrm{H}_{2} \mathrm{O}_{2}$ treatment, RGC-5 cell viability, oxidative stress, apoptosis, and autophagy were determined. As shown in Figure $2 B-2 G$, miR-155 inhibition protected RGC-5 cells from $\mathrm{H}_{2} \mathrm{O}_{2}$-induced injury.

\section{Acteoside attenuates $\mathrm{H}_{2} \mathrm{O}_{2}$-induced $\mathrm{RGC}-5$ cell injury via modulating miR-155}

Our previous study indicated that acteoside was involved in glaucoma treatment and could inhibit autophagic apoptosis of RGC-5 cells (4). We next explored the relationship between acteoside and miR-155. Acteoside administration decreased miR-155 expression in $\mathrm{H}_{2} \mathrm{O}_{2}$-treated RGC-5 cells (Figure $3 A$ ). We also overexpressed miR-155 via miR-155 mimic transfection, and NC mimic was the corresponding control (Figure 3B). miR-155 overexpression abolished the protective effect of acteoside on cell viability, oxidative stress, apoptosis, and autophagy (Figure $3 C-3 H$ ). These findings demonstrated that acteoside attenuates $\mathrm{H}_{2} \mathrm{O}_{2}$ induced RGC-5 cell oxidative stress and injury via miR-155. 

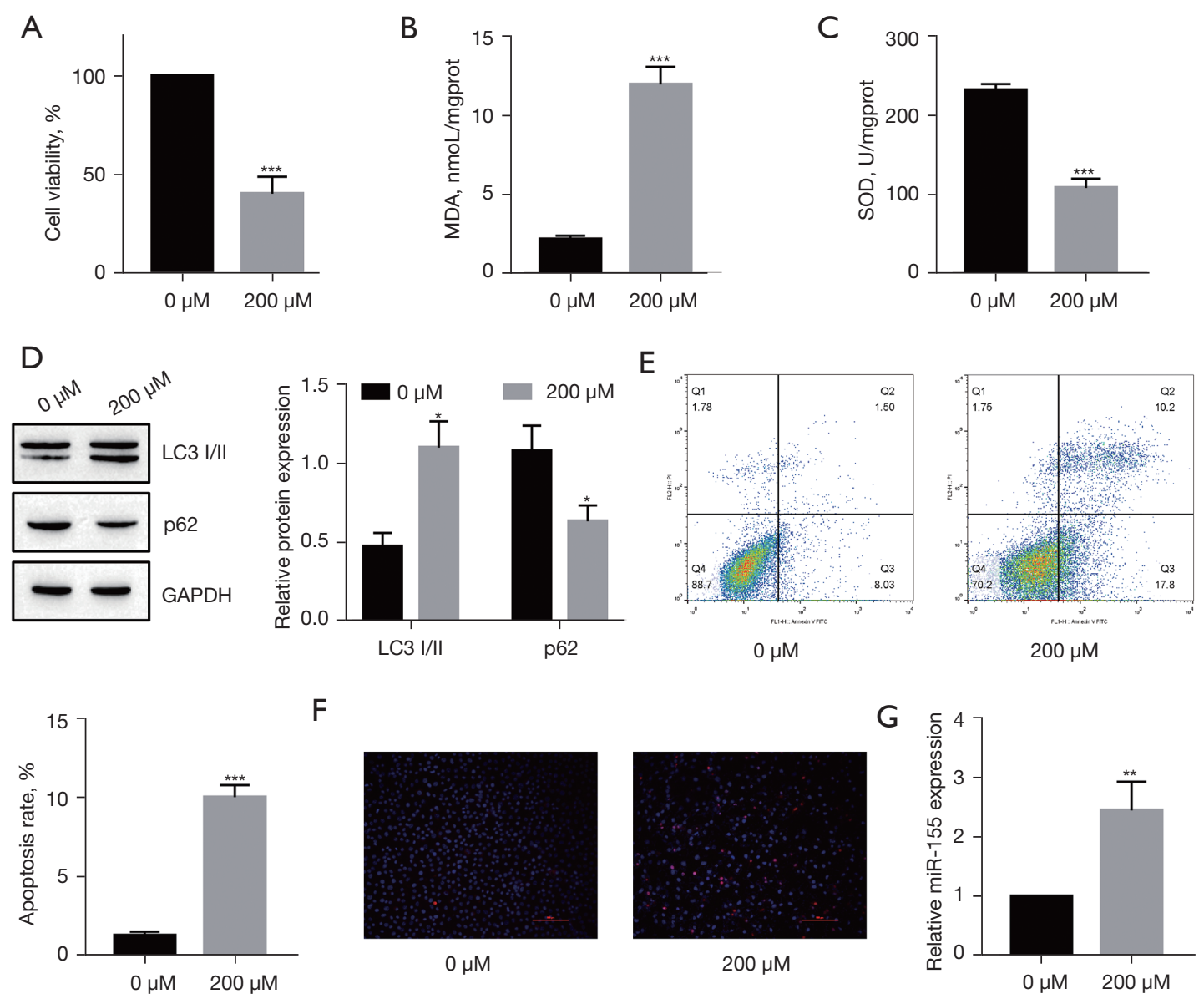

Figure 1 MiR-155 is induced upon $\mathrm{H}_{2} \mathrm{O}_{2}$ treatment of RGC-5 cells. (A) Cell viability of $0 \mu M$ - or $200 \mu \mathrm{M}$-treated RGC-5 cells was measured by the MTT assay; (B) MDA content; (C) SOD activity; (D) expression of autophagic proteins in $0 \mu M-$ or $200 \mu M$-treated RGC-5 cells was measured by western blot; (E) apoptosis of $0 \mu \mathrm{M}$ - and $200 \mu \mathrm{M}$-treated RGC-5 cells was measured by flow cytometry; (F) TUNEL staining of RGC-5 cells (scale bar $=200 \mu \mathrm{m}$ ); (G) expression of miR-155 in $0 \mu \mathrm{M}$ - and $200 \mu M$-treated RGC-5 cells was measured by $\mathrm{RT}-\mathrm{qPCR} .{ }^{*} \mathrm{P}<0.05 ;{ }^{*} \mathrm{P}<0.01 ;{ }^{* *} \mathrm{P}<0.001$. MDA, malondialdehyde; SOD, superoxide dismutase.

\section{LncRNA CASC2 targets miR-155 in RGC-5 cells}

The bioinformatics software starBase (http://starbase.sysu. edu.cn/) was used to identify potential targets of miR-155, and lncRNA CASC2 was selected due to its role in acteoside attenuates $\mathrm{H} 2 \mathrm{O} 2$-induced RGC-5 cell injury. The binding sites between miR-155 and WT or MUT CASC2 3'-UTR are shown in Figure 4A. The expression of CASC2 in $\mathrm{H}_{2} \mathrm{O}_{2}$-induced RGC-5 cells was subsequently analyzed, its expression had a significantly downregulated (Figure 4B). To confirm whether miR-155 could bind to CASC2 3'UTR, a luciferase reporter vector containing the WT or MUT 3'-UTR of CASC2 was constructed. Overexpression of miR-155 markedly inhibited the luciferase activity of the WT reporter vector but did not affect the luciferase activity of the mutant reporter vector in RGC-5 cells (Figure 4C). Furthermore, overexpression of miR-155 also significantly suppressed CASC2 expression (Figure 4D). These results suggested that miR-155 directly targeted the CASC2 gene.

\section{Acteoside attenuates $\mathrm{H}_{2} \mathrm{O}_{2}$-induced $\mathrm{RGC}-5$ cell injury via modulating CASC2/miR-155 axis}

We also overexpressed CASC2 via CASC2 overexpression vector oe-CASC2 (Figure 5A). The results indicated that acteoside attenuates $\mathrm{H}_{2} \mathrm{O}_{2}$-induced RGC-5 cell oxidative stress and injury, which was reversed with overexpression of miR-155, whereas the overexpression of CASC2 repressed the effect of acteoside on the cell viability, oxidative stress, apoptosis, and autophagy (Figure $5 B-5 G$ ). These findings 
A

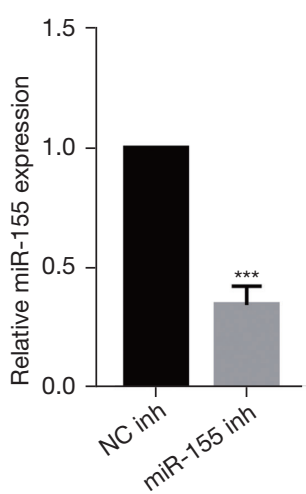

$\mathrm{E}$

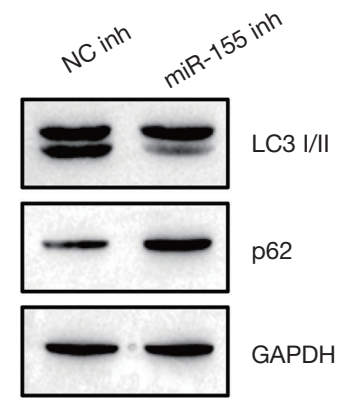

B

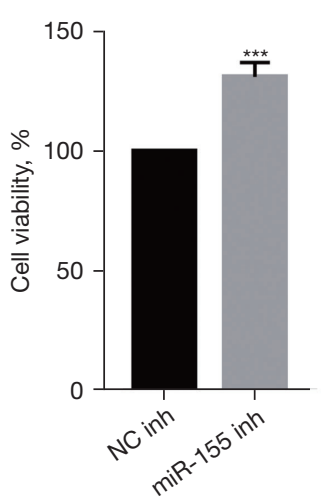

C

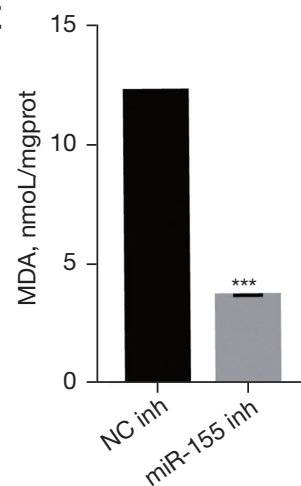

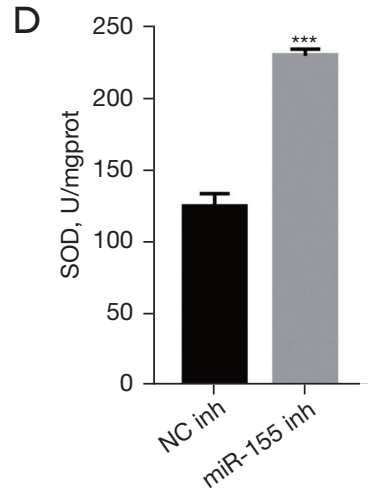

$\mathrm{F}$
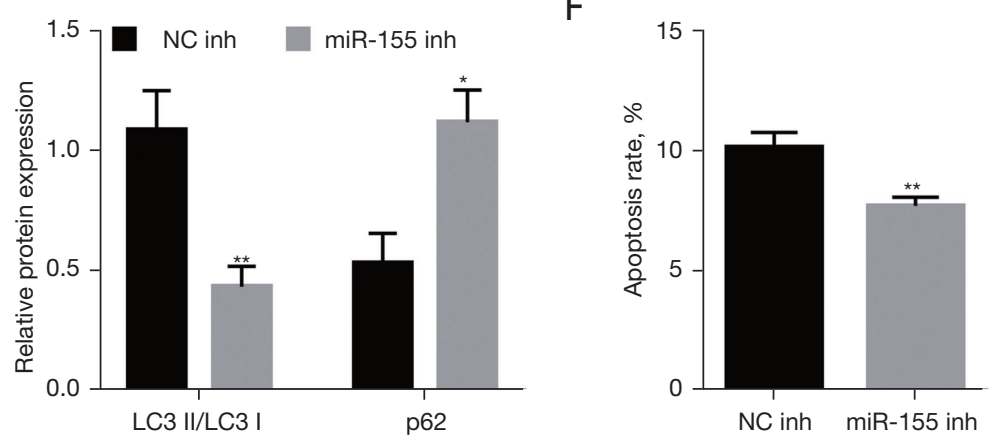
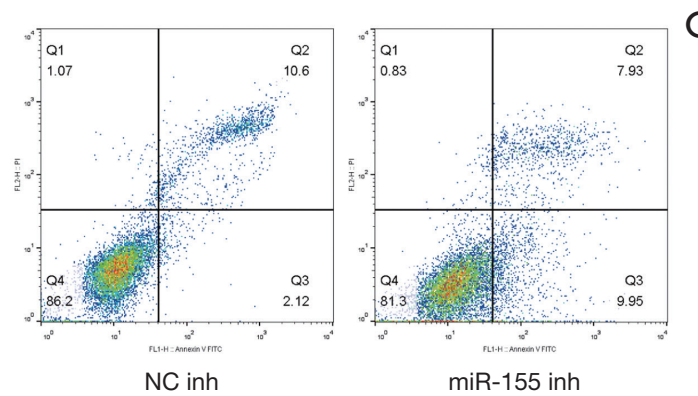

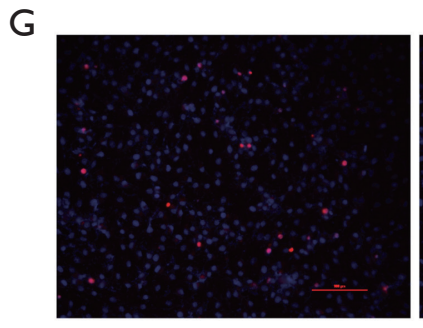

NC inh

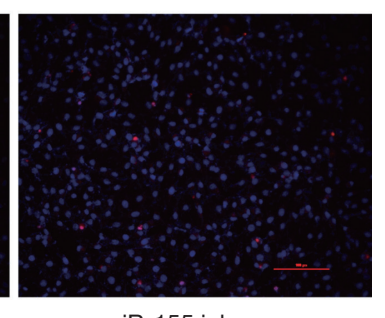

miR-155 inh

Figure 2 MiR-155 inhibition alleviates $\mathrm{H}_{2} \mathrm{O}_{2}$-induced RGC-5 cell injury. (A) Expression of miR-155was measured by RT-qPCR; (B) the cell viability was measured by the MTT assay; (C) MDA content was measured by ELISA; (D) SOD activity was measured by ELISA; (E) the expression of autophagic proteins was measured western blot; (F) apoptosis of RGC-5 cells was measured by flow cytometry; (G) TUNEL staining of RGC-5 cells. Scale bar $=200 \mu \mathrm{m}$. ${ }^{*} \mathrm{P}<0.05 ;{ }^{* *} \mathrm{P}<0.01 ;{ }^{* * *} \mathrm{P}<0.001$. RT-qPCR, quantitative real-time PCR; MDA, malondialdehyde; SOD, superoxide dismutase.

demonstrated that acteoside attenuates $\mathrm{H}_{2} \mathrm{O}_{2}$-induced RGC5 cell oxidative stress and injury via CASC $2 / \mathrm{miR}-155$ axis.

\section{Acteoside protects $\mathrm{RGC}-5$ cells against $\mathrm{H}_{2} \mathrm{O}_{2}$-induced cell injury via the miR-155/mTOR axis}

Given that miR-155 functions via mTOR signaling (22) and mTOR was involved in RGC damage and glaucoma development (8), we next determined the role of mTOR in the protective effect of acteoside/miR-155 on RGC-5 cells. It was found that mTOR expression was decreased in $\mathrm{H}_{2} \mathrm{O}_{2}$ treated RGC-5 cells (Figure 6A), and miR-155 inhibition could induce mTOR expression (Figure $6 B$ ). In addition, acteoside administration promoted mTOR expression while miR-155 overexpression abolished the promotive effect of acteoside on mTOR expression (Figure 6C). We further employed an mTOR agonist and inhibitor, HY-B0795 and rapamycin, to determine the relationship between acteoside 
A

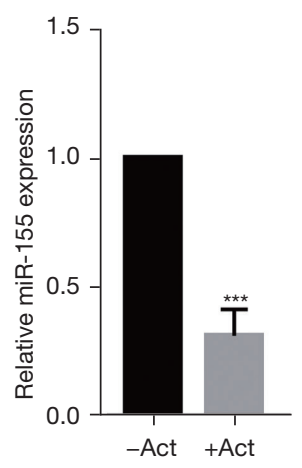

E

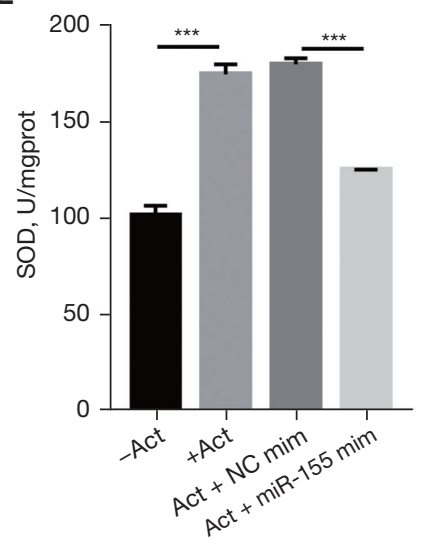

B

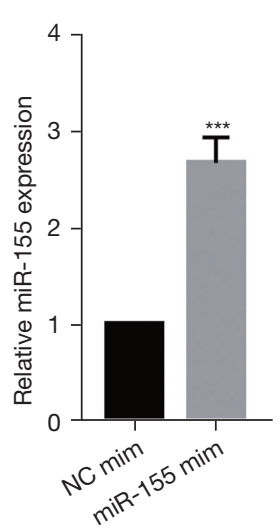

$\mathrm{F}$
C

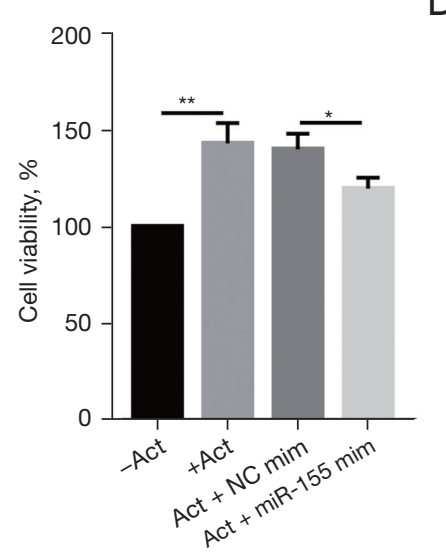

D

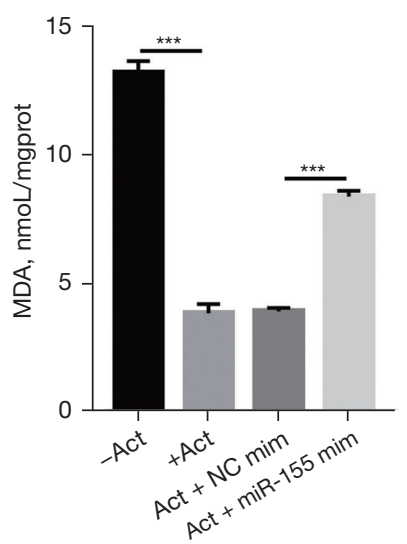

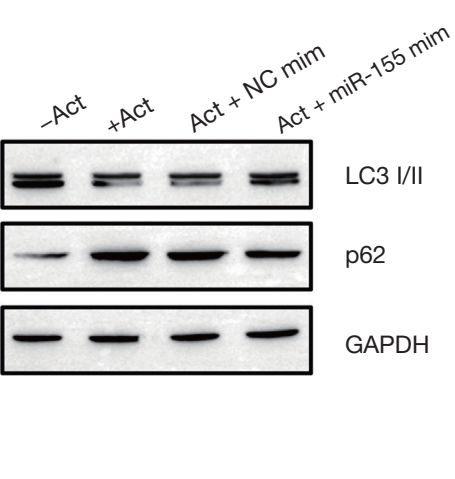

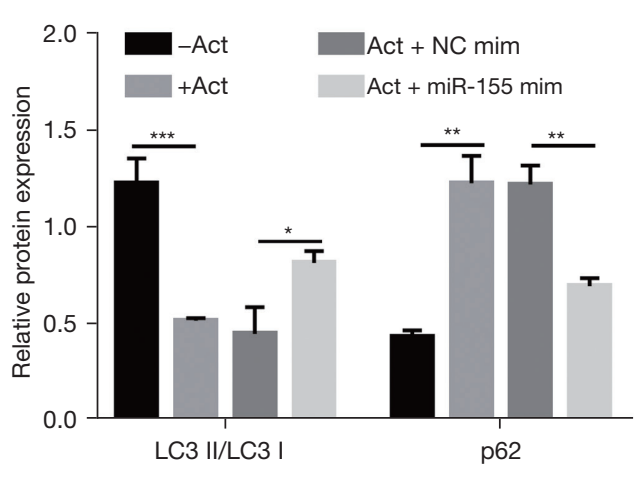

G
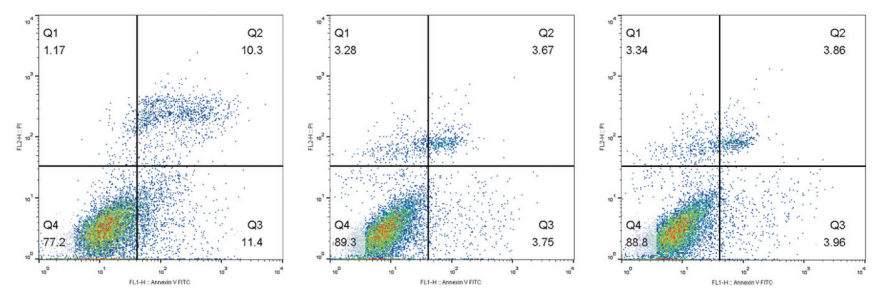

$\mathrm{H}$

Act
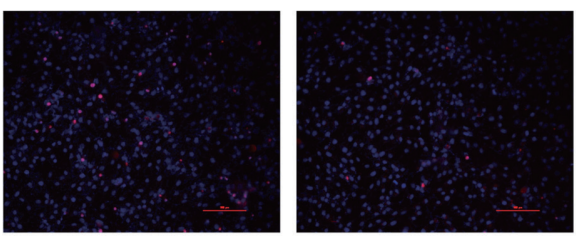

Act $+\mathrm{NC}$ mim

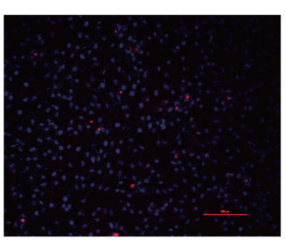

Act $+\mathrm{NC}$ mim

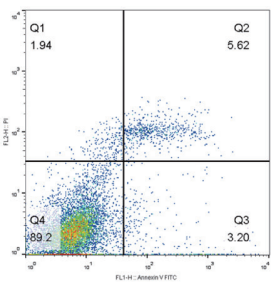

Act $+\mathrm{miR}-155 \mathrm{mim}$
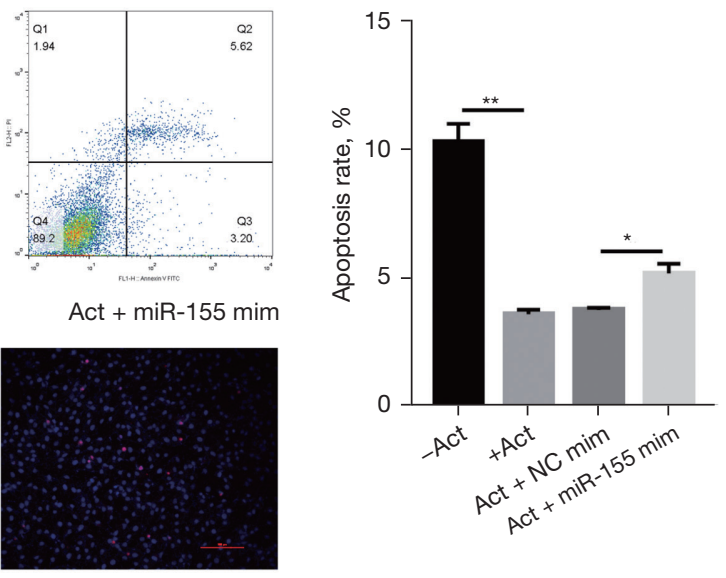

Act $+\mathrm{miR}-155 \mathrm{mim}$

Figure 3 Acteoside attenuates $\mathrm{H}_{2} \mathrm{O}_{2}$-induced RGC-5 cell injury via modulating miR-155. (A) Expression of miR-155 in acteoside-treated RGC-5 cells was measured by RT-qPCR; (B) expression of miR-155 in RGC-5 cells was measured by RT-qPCR. (C) the viability of RGC5 cells was measured by the MTT assay; (D) MDA content was measured by ELISA; (E) SOD activity was measured by ELISA; (F) the expression of autophagic proteins in RGC-5 cells was measured by western blot; (G) apoptosis of RGC-5 cells was measured by flow cytometry; (H) TUNEL staining of RGC-5 cells. Scale bar $=200 \mu \mathrm{m}$. ${ }^{*} \mathrm{P}<0.05 ;{ }^{* *} \mathrm{P}<0.01$; ${ }^{* * *} \mathrm{P}<0.001$. RT-qPCR, quantitative real-time PCR; MDA, malondialdehyde; SOD, superoxide dismutase. 
A

LnCCASC2-WT: 5'-UCUACACCCACAAAAAGCAUUAU-3'

$1|1| 1 \mid$

miR-151: 3'-UGGGGAUAGUGCUAAUCGUAAUU-5'

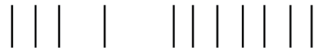

LnCCASC2-MUT: 5'-UCUACACCCACAAAAUCGUAAUU-3'

B

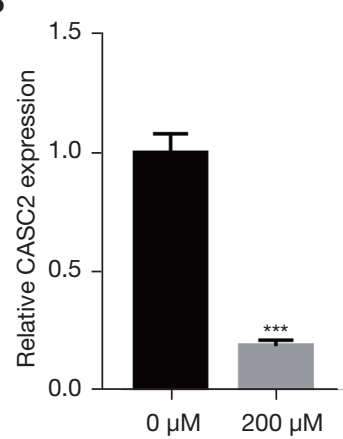

C

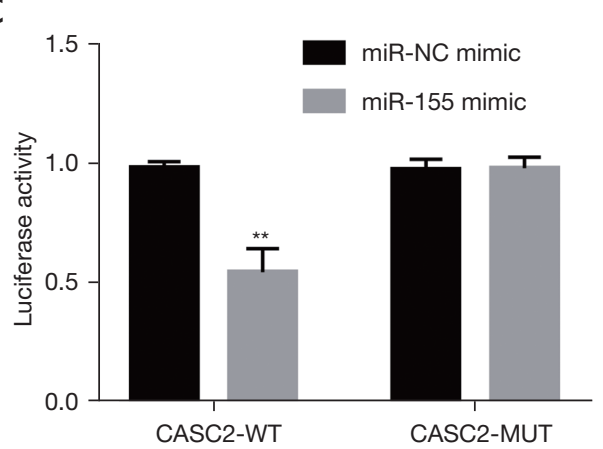

D

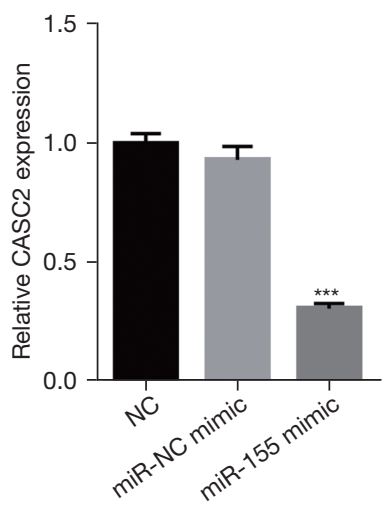

Figure 4 CASC2 targets miR-155 in RGC-5 cells. (A) Schematic representation of the binding site of miR-155 in CASC2-WT and in CASC2-MUT; (B) RT-qPCR was used to determine the expression of CASC2; (C) dual-luciferase reporter assays were used to demonstrate that CASC2 can target miR-155; (D) RT-qPCR was used to determine the expression of CASC2. ${ }^{* *} \mathrm{P}<0.01$; ${ }^{* *} \mathrm{P}<0.001$. RT-qPCR, quantitative real-time PCR.

and mTOR. As shown in Figure 6D-6I, HY-B0795 and rapamycin promoted and reduced the protective effect of acteoside on attenuating RGC injury, respectively. Thus, these findings suggest that acteoside protects RGC-5 cells against $\mathrm{H}_{2} \mathrm{O}_{2}$-induced cell injury via miR-155/mTOR.

\section{Discussion}

Glaucoma is the second leading cause of blindness worldwide, which is characterized by optic nerve atrophy and visual field defects. It is believed that RGC injury is the main cause of glaucoma $(23,24)$. It is reported that miRNAs could be promising biomarkers for glaucoma, and aberrant expression of miRNAs is involved in glaucoma development and treatment (6,25). For example, miR-93-5p is downregulated in NMDA-induced RGCs and a glaucoma rat model, and miR-93-5p overexpression suppresses RGC autophagy in NMDA-induced glaucoma (8). miR-200a is decreased in glaucoma and plays a protective role in improving glaucoma-induced optical nerve injury (26). Furthermore, miR-149 downregulation suppresses RGC apoptosis in glaucoma mice (10), and a previous study showed that miR-155 is upregulated in glaucoma (11). Notably, the expression of miR-155 is upregulated in the $\mathrm{H}_{2} \mathrm{O}_{2}$-induced cells, including vascular smooth muscle cells (27), human umbilical vein endothelial cells (28), and human embryonic kidney 293 cells (29), and then silencing of miR-155 can attenuate $\mathrm{H}_{2} \mathrm{O}_{2}$-induced cell injury (27). Here, we established RGC injury via $200 \mu \mathrm{M}$ $\mathrm{H}_{2} \mathrm{O}_{2}$ administration and found that miR-155 was induced in $\mathrm{H}_{2} \mathrm{O}_{2}$-treated RGCs. Thus, the miR-155 was investigated in subsequent assays. Moreover, inhibition of miR-155 promoted RGC viability while repressing RGC apoptosis and autophagy. Emerging studies have paid much attention to the therapeutic effects of Chinese traditional medicine on glaucoma $(2,30)$. Our previous work indicates that acteoside, which is the effective component of Yunnan Kudingcha, plays a critical role in glaucoma via inhibiting autophagy-induced apoptosis in RGCs (4). Here, we found that acteoside inhibits miR155 expression in $\mathrm{H}_{2} \mathrm{O}_{2}$-treated RGCs. Importantly, miR-155 overexpression inhibits the protective effect of acteoside on attenuating RGC injury, but overexpression of CASC2 finally reverses the inhibitor effect of miR-155 overexpression on $\mathrm{H}_{2} \mathrm{O}_{2}$-treated RGCs.

Although miRNAs play roles in glaucoma, the involved mechanisms are different. Zhang et al. suggested that miR141-3p inhibits retinal neovascularization in glaucoma mice via the DOK5-mediated MAPK signaling pathway (9). Su et al. demonstrated that miR-21a-5p modulates 

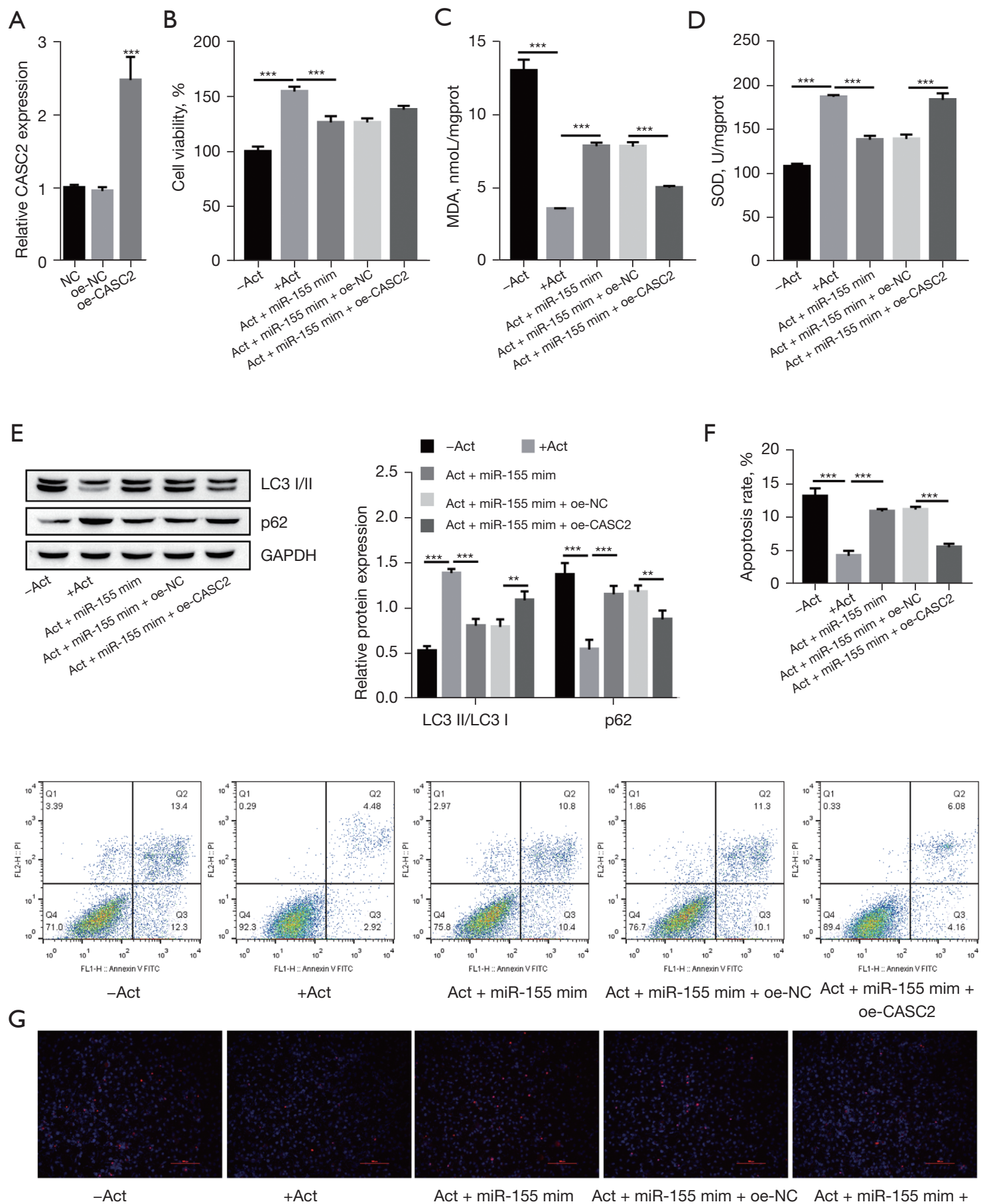

Act + miR-155 mim

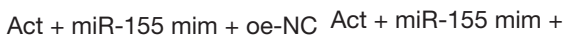

-Act
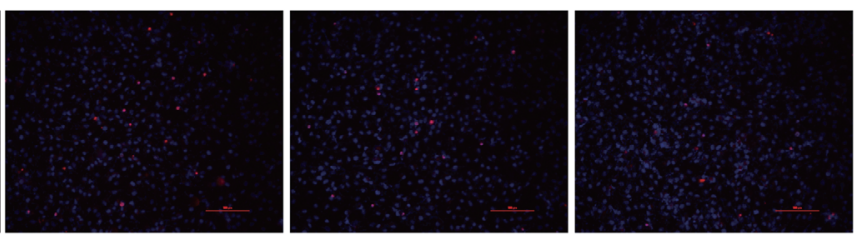

Act $+\mathrm{miR}-155 \mathrm{mim} \quad$ Act $+\mathrm{miR}-155 \mathrm{mim}+$ oe-NC Act $+\mathrm{miR}-155 \mathrm{mim}+$ oe-CASC2

Figure 5 Acteoside attenuates $\mathrm{H}_{2} \mathrm{O}_{2}$-induced RGC-5 cell injury via modulating CASC2/miR-155 axis. (A) Expression of miR-155 in oeCASC2-transfected RGC-5 cells was measured by RT-qPCR; (B) the viability of RGC-5 cells was measured by the MTT assay; (C) MDA content was measured by ELISA; (D) SOD activity was measured by ELISA; (E) the expression of autophagic proteins in RGC-5 cells was measured by western blot; (F) apoptosis of RGC-5 cells was measured by flow cytometry; (G) TUNEL staining of RGC-5 cells. Scale bar = $200 \mu \mathrm{m} .{ }^{*} \mathrm{P}<0.01 ;{ }^{* *} \mathrm{P}<0.001$. RT-qPCR, quantitative real-time PCR; MDA, malondialdehyde; SOD, superoxide dismutase. 
A
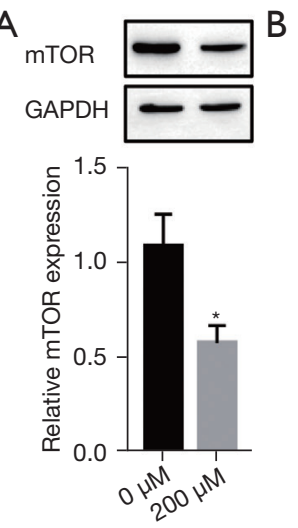

$\mathrm{E}$

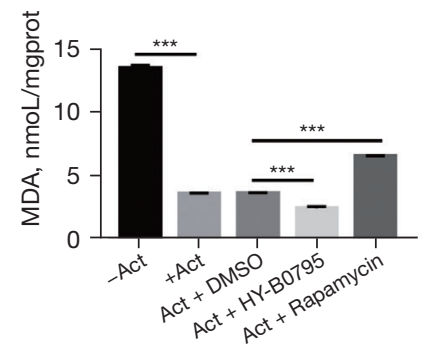

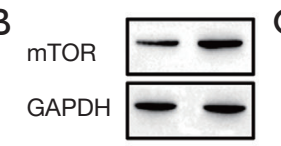

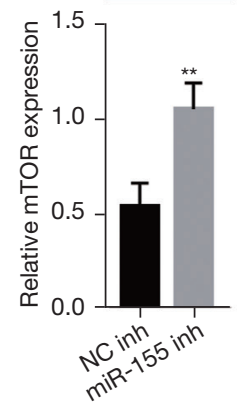

$\mathrm{F}$

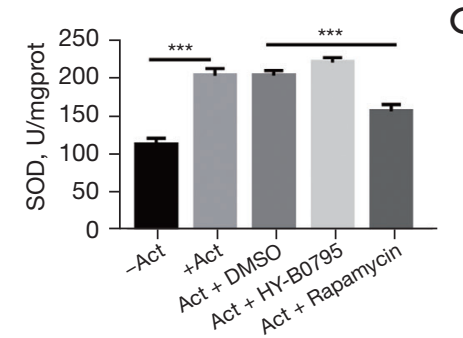

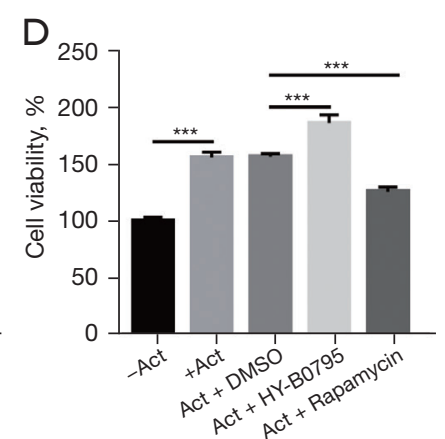

G

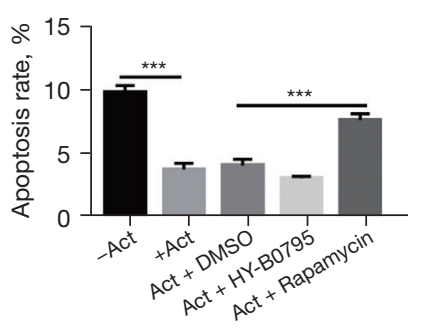

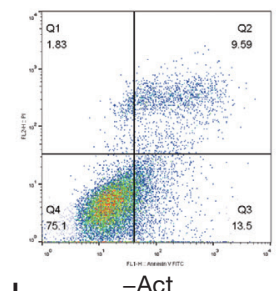

$\mathrm{H}$
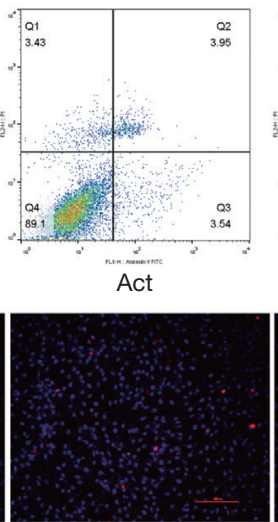

Act

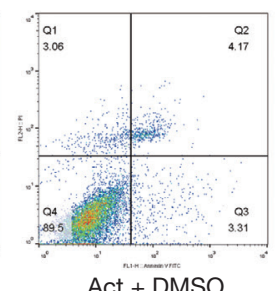

Act + DMSO

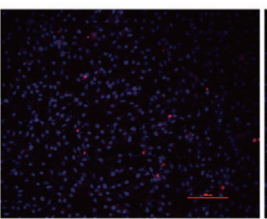

Act + DMSO

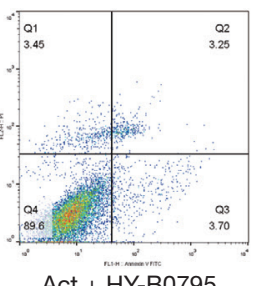

Act + HY-B0795

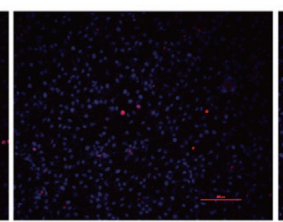

Act + HY-B0795

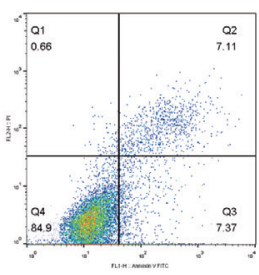

Act + Rapamycin

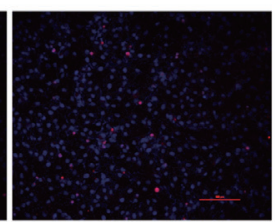

Act + Rapamycin

I
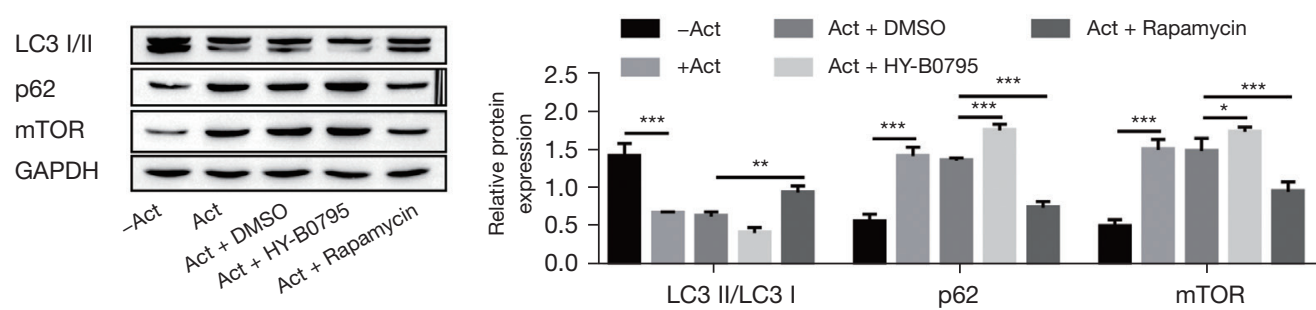

Figure 6 Acteoside protects RGC-5 cells against H2O2-induced cell injury via the miR-155/mTOR axis. (A) The expression of mTOR in $0 \mu \mathrm{M}$ - or $200 \mu \mathrm{M}$-treated RGC-5 cells was measured by western blot; (B) the expression of mTOR in RGC-5 cells was measured by western blot; (C) the expression of mTOR in RGC-5 cells was measured by western blot; (D) the viability of RGC-5 cells was measured by the MTT assay; (E) MDA content was measured by ELISA; (F) SOD activity was measured by ELISA; (G) apoptosis of RGC-5 cells was measured by flow cytometry; (H) TUNEL staining of RGC-5 cells; (I) the expression of mTOR and autophagic proteins in RGC-5 cell were measured by western blot. Scale bar $=200 \mu \mathrm{m} .{ }^{*} \mathrm{P}<0.05 ;{ }^{* *} \mathrm{P}<0.01 ;{ }^{* * *} \mathrm{P}<0.001$. MDA, malondialdehyde; SOD, superoxide dismutase. 
mesenchymal stem cell-mediated neuroprotective effects on acute glaucoma via targeting PDCD4 (31). Several studies show that the mTOR signaling pathway is involved in miR-155-mediated regulation $(16,22)$. In addition, mTOR contributes to glaucoma development and is associated with RGC activity $(13,32,33)$. Here, we found that the expression of mTOR was downregulated in $\mathrm{H}_{2} \mathrm{O}_{2}$ treated RGCs. Acteoside administration or miR-155 inhibition increased mTOR expression. However, miR-155 overexpression abolished the promotive effect of acteoside on the expression of mTOR. The mTOR inhibitor rapamycin reversed the protective effect of acteoside on the attenuation of RGC injury. Taken together, our findings demonstrate that acteoside attenuates RGC injury via the $\mathrm{miR}-155 / \mathrm{mTOR}$ axis, which is of significance for glaucoma treatment.

\section{Conclusions}

We report that acteoside repressed RGC injury via targeting the CASC $2 / \mathrm{miR}-155 / \mathrm{mTOR}$ axis. The expression of miR-155 was induced in $\mathrm{H}_{2} \mathrm{O}_{2}$-treated RGCs. Induced miR-155 expression promoted RGC apoptosis and autophagy. Acteoside administration could suppress miR155 expression and miR-155 overexpression abolished the protective effect of acteoside on RGCs. The expression of CASC2 was decreased in $\mathrm{H}_{2} \mathrm{O}_{2}$-treated RGCs. Acteoside administration could increase CASC2 expression and CASC2 overexpression reverses the effect of miR-155 overexpression on acteoside treatment-RGCs. Enhanced miR-155 repressed the mTOR signaling pathway while acteoside activated the mTOR signaling pathway. The mTOR inhibitor rapamycin rescued the protective effect of acteoside on RGCs. Therefore, our study describes a novel mechanism by which acteoside protects RGC-5 cells against $\mathrm{H}_{2} \mathrm{O}_{2}$-induced cell injury via the CASC2/miR-155/ mTOR axis.

\section{Acknowledgments}

Funding: This work was supported by Provincial Basic Research Plan, Kunming Medical Joint Special Project [2017FE468(-175)]; Provincial Basic Research Plan, Kunming Medical Joint Special Project [2017FE468(-046)]; Scientific Research Projects of Research Institutions in Yunnan Medical and Health Units (2018NS0145); Bethune Langmu Middle-aged and Youth Ophthalmology Research Fund (BJ-LM2017006L); National Natural Science
Foundation of China (82060178); Doctoral Research Fund Project of The First Affiliated Hospital of Kunming Medical University (2020BS0022); Doctoral Innovation Fund Project of Kunming Medical University.

\section{Footnote}

Reporting Checklist: The authors have completed the MDAR reporting checklist. Available at https://dx.doi. org/10.21037/atm-21-5630

Data Sharing Statement: Available at https://dx.doi. org/10.21037/atm-21-5630

Conflicts of Interest: All authors have completed the ICMJE uniform disclosure form (available at https://dx.doi. org/10.21037/atm-21-5630). The authors have no conflicts of interest to declare.

Ethical Statement: The authors are accountable for all aspects of the work in ensuring that questions related to the accuracy or integrity of any part of the work are appropriately investigated and resolved.

Open Access Statement: This is an Open Access article distributed in accordance with the Creative Commons Attribution-NonCommercial-NoDerivs 4.0 International License (CC BY-NC-ND 4.0), which permits the noncommercial replication and distribution of the article with the strict proviso that no changes or edits are made and the original work is properly cited (including links to both the formal publication through the relevant DOI and the license). See: https://creativecommons.org/licenses/by-nc-nd/4.0/.

\section{References}

1. Madeira MH, Ortin-Martinez A, Nadal-Nícolas F, et al. Caffeine administration prevents retinal neuroinflammation and loss of retinal ganglion cells in an animal model of glaucoma. Sci Rep 2016;6:27532.

2. Mi XS, Zhong JX, Chang RC, et al. Research advances on the usage of traditional Chinese medicine for neuroprotection in glaucoma. J Integr Med 2013;11:233-40.

3. Li X, Wang Q, Ren Y, et al. Tetramethylpyrazine protects retinal ganglion cells against $\mathrm{H} 2 \mathrm{O} 2$-induced damage via the microRNA-182/mitochondrial pathway. Int J Mol Med 2019;44:503-12.

4. Chen Q, Xi X, Zeng Y, et al. Acteoside inhibits 
autophagic apoptosis of retinal ganglion cells to rescue glaucoma-induced optic atrophy. J Cell Biochem 2019;120:13133-40.

5. Li T, Wang Y, Chen J, et al. Co-delivery of brinzolamide and miRNA-124 by biodegradable nanoparticles as a strategy for glaucoma therapy. Drug Deliv 2020;27:410-21.

6. Hindle AG, Thoonen R, Jasien JV, et al. Identification of Candidate miRNA Biomarkers for Glaucoma. Invest Ophthalmol Vis Sci 2019;60:134-46.

7. Guo R, Shen W, Su C, et al. Relationship between the Pathogenesis of Glaucoma and miRNA. Ophthalmic Res 2017;57:194-9.

8. Li R, Jin Y, Li Q, et al. MiR-93-5p targeting PTEN regulates the NMDA-induced autophagy of retinal ganglion cells via AKT/mTOR pathway in glaucoma. Biomed Pharmacother 2018;100:1-7.

9. Zhang LQ, Cui H, Yu YB, et al. MicroRNA-141-3p inhibits retinal neovascularization and retinal ganglion cell apoptosis in glaucoma mice through the inactivation of Docking protein 5-dependent mitogen-activated protein kinase signaling pathway. J Cell Physiol 2019;234:8873-87.

10. Nie XG, Fan DS, Huang YX, et al. Downregulation of microRNA-149 in retinal ganglion cells suppresses apoptosis through activation of the PI3K/Akt signaling pathway in mice with glaucoma. Am J Physiol Cell Physiol 2018;315:C839-49.

11. Juźwik CA, S Drake S, Zhang Y, et al. microRNA dysregulation in neurodegenerative diseases: A systematic review. Prog Neurobiol 2019;182:101664.

12. Aggio-Bruce R, Chu-Tan JA, Wooff Y, et al. Inhibition of microRNA-155 Protects Retinal Function Through Attenuation of Inflammation in Retinal Degeneration. Mol Neurobiol 2021;58:835-54.

13. Madrakhimov SB, Yang JY, Kim JH, et al. mTORdependent dysregulation of autophagy contributes to the retinal ganglion cell loss in streptozotocin-induced diabetic retinopathy. Cell Commun Signal 2021;19:29.

14. Liu S, Chen S, Ren J, et al. Ghrelin protects retinal ganglion cells against rotenone via inhibiting apoptosis, restoring mitochondrial function, and activating AKTmTOR signaling. Neuropeptides 2018;67:63-70.

15. Li Z, Shen D, Meng Y, et al. miR-155-5p alleviates ethanol-induced myocardial insulin resistance in $\mathrm{H} 9 \mathrm{C} 2$ cells via regulating the mTOR signalling pathway. Mol Biol Rep 2020;47:9469-77.

16. Wang F, Shan S, Huo Y, et al. MiR-155-5p inhibits PDK1 and promotes autophagy via the mTOR pathway in cervical cancer. Int J Biochem Cell Biol 2018;99:91-9.
17. Babuta M, Furi I, Bala S, et al. Dysregulated Autophagy and Lysosome Function Are Linked to Exosome Production by Micro-RNA 155 in Alcoholic Liver Disease. Hepatology 2019;70:2123-41.

18. Kang Y, Jia P, Zhao H, et al. MicroRNA-26a overexpression protects RGC-5 cells against $\mathrm{H} 2 \mathrm{O} 2$ induced apoptosis. Biochem Biophys Res Commun 2015;460:164-9.

19. Tang, Z., Xia, Y., Chen, Q., Li, Y., He, Z., Zhao, X., 2015. The effects of different concentrations of Acteoside on the RGC cell model in vitro. Chin J Hypertens 23, 1.

20. Livak KJ, Schmittgen TD. Analysis of relative gene expression data using real-time quantitative PCR and the 2(-Delta Delta C(T)) Method. Methods 2001;25:402-8.

21. Zhao W, Wang S, Qin T, et al. Arbutin attenuates hydrogen peroxide-induced oxidative injury through regulation of microRNA-29a in retinal ganglion cells. Biomed Pharmacother 2019;112:108729.

22. Li Z, Hu Z, Meng Y, et al. miR-155-5p upregulation ameliorates myocardial insulin resistance via mTOR signaling in chronic alcohol drinking rats. PeerJ 2021;9:e10920.

23. Russo R, Varano GP, Adornetto A, et al. Retinal ganglion cell death in glaucoma: Exploring the role of neuroinflammation. Eur J Pharmacol 2016;787:134-42.

24. Shen J, Wang Y, Yao K. Protection of retinal ganglion cells in glaucoma: Current status and future. Exp Eye Res 2021;205:108506.

25. Molasy M, Walczak A, Szaflik J, et al. MicroRNAs in glaucoma and neurodegenerative diseases. J Hum Genet 2017;62:105-12.

26. Peng H, Sun YB, Hao JL, et al. Neuroprotective effects of overexpressed microRNA-200a on activation of glaucomarelated retinal glial cells and apoptosis of ganglion cells via downregulating FGF7-mediated MAPK signaling pathway. Cell Signal 2019;54:179-90.

27. Zhao L, Ouyang Y, Bai Y, et al. miR-155-5p inhibits the viability of vascular smooth muscle cell via targeting FOS and ZIC3 to promote aneurysm formation. Eur J Pharmacol 2019;853:145-52.

28. Choi S, Kim J, Kim JH, et al. Carbon monoxide prevents TNF- $\alpha$-induced eNOS downregulation by inhibiting NF$\kappa \mathrm{B}-$ responsive miR-155-5p biogenesis. Exp Mol Med 2017;49:e403.

29. Huang LG, Zou J, Lu QC. Silencing rno-miR$155-5 \mathrm{p}$ in rat temporal lobe epilepsy model reduces pathophysiological features and cell apoptosis by activating Sestrin-3. Brain Res 2018;1689:109-22. 
30. Yu N, Zhang Z, Chen P, et al. Tetramethylpyrazine (TMP), an Active Ingredient of Chinese Herb Medicine Chuanxiong, Attenuates the Degeneration of Trabecular Meshwork through SDF-1/CXCR4 Axis. PLoS One 2015;10:e0133055.

31. Su W, Li Z, Jia Y, et al. microRNA-21a-5p/PDCD4 axis regulates mesenchymal stem cell-induced neuroprotection in acute glaucoma. J Mol Cell Biol 2017;9:289-301.

32. Teotia P, Van Hook MJ, Fischer D, et al. Human retinal ganglion cell axon regeneration by recapitulating

Cite this article as: Xi X, Ma J, Chen Q, Wang X, Xia Y, Wen X, Yuan J, Li Y. Acteoside attenuates hydrogen peroxide-induced injury of retinal ganglion cells via the CASC2/miR-155/mTOR

axis. Ann Transl Med 2022;10(1):5. doi: 10.21037/atm-21-5630 developmental mechanisms: effects of recruitment of the mTOR pathway. Development 2019;146:dev178012.

33. Harder JM, Guymer C, Wood JPM, et al. Disturbed glucose and pyruvate metabolism in glaucoma with neuroprotection by pyruvate or rapamycin. Proc Natl Acad Sci U S A 2020;117:33619-27.

(English Language Editor: C. Betlazar-Maseh) 